\title{
Hepatotoxicity and Anti-Nuclear Antibody Positivity During Methimazole Therapy for Thyrotoxicosis: A Case Report
}

\author{
Ali Gokyer ${ }^{\mathrm{a}, \mathrm{d}}$, Mehmet Celik ${ }^{\mathrm{b}}$, Huseyin Celik ${ }^{\mathrm{b}}$, Semra Ayturk ${ }^{\mathrm{b}}$, Sibel Guldiken ${ }^{\mathrm{b}}$, Armagan Tugrul ${ }^{\mathrm{b}}$, \\ Ayten Ustundag ${ }^{\mathrm{c}}$, Ahmet Tezel ${ }^{\mathrm{c}}$
}

\begin{abstract}
Thyrotoxicosis is one of the most frequent endocrine disorders. Methimazole is the anti-thyroid drug for the treatment of hyperthyrodism. Methimazole is rarely associated with hepatic toxicity. We reported a case of 56-year-old woman who developed hepatotoxicity and antinuclear antibody positivity during methimazole treatment. Increased liver enzyme levels appeared after 1 month of the methimazole treatment. Viral hepatitis markers were negative. The radiological examinations were normal. According to the CIOMS/RUCAM scale, the score was 11 and the causal relationship of the hepatic adverse reaction by methimazole was highly probable. Methimazole-induced hepatotoxicity was considered and methimazole treatment was cancelled. The laboratory parameters returned to normal after 15 days. The mechanism of hepatotoxicity due to methimazole treatment was not fully understood. It has been hypothesized that there is a genetic predisposition, associated with a dose-dependent immune reaction. In conclusion, physicians should be aware the risk of hepatotoxicity related with methimazole treatment.
\end{abstract}

Keywords: Hepatotoxicity; Methimazole; Thyrotoxicosis

\section{Introduction}

Thyrotoxicosis is a common condition associated with excess circulating thyroid hormones free thyroxine (FT4) and/or free triiodothyronine (FT3) [1]. The term thyrotoxicosis, rather

Manuscript accepted for publication December 02, 2014

aDepartment of Internal Medicine, Trakya Medical School, University of Trakya, Edirne, Turkey

bepartment of Internal Medicine, Division of Endocrinology and Metabolism, Trakya Medical School, University of Trakya, Edirne, Turkey

${ }^{\mathrm{c}}$ Department of Internal Medicine, Division of Gastroenterology, Trakya Medical School, University of Trakya, Edirne, Turkey

${ }^{\mathrm{d} C}$ Corresponding Author: Ali Gokyer, Department of Internal Medicine, Trakya

University Medical Faculty, Edirne 22030, Turkey.

Email: aligkyer@hotmail.com

doi: http://dx.doi.org/10.14740/jmc2009w than hyperthyroidism, should be used for this disorder, because it needs not to be associated with hyperfunction of the thyroid gland. The term hyperthyroidism is reserved for disorders that result from sustained overproduction and release of hormone by the thyroid gland. Current treatment of hyperthyroidism is based on drugs, surgery and radioactive iodine.

Methimazole is the anti-thyroid drug of choice because of its potency and infrequent side effects which are usually mild [2]. Methimazole is associated with cholestasis rather than hepatic necrosis, and the risk is increased with higher doses administered in older patients. Mild side effects occur in up to $20 \%$ of patients and include skin reactions, arthralgia, gastrointestinal symptoms, an abnormal sense of taste, and occasionally, sialadenitis. Other effects include thrombocytopenia, enlargement of lymph nodes, a lupus-like syndrome including the development of anti-neutrophil cytoplasmic antibody (ANCA)-positive vasculitis and toxic psychoses [3].

In this report, a case is presented with thyrotoxicosis and treated with methimazole, but the patient developed hepatotoxicity and anti-nuclear antibody (anti-ANA) positivity.

\section{Case Report}

A 56-year-old woman with a 2-month history of sweating and palpitations was admitted to the hospital.

Biochemical values were compatible with primary hyperthyroidism. Pre-treatment liver enzyme values were normal. She was taking the methimazole tb $(30 \mathrm{mg} /$ day $)$ and nebivolol tb $(5 \mathrm{mg} /$ day $)$ for 1 week. The liver enzyme values increased at the end of the first month of methimazol treatment (Table 1). Patient was referred to our department with increased liver enzymes, normal upper abdominal MRI and MR cholangiography. Patient was admitted to our outpatient clinic with the symptom of weakness. On physical examination, BP was $120 / 80 \mathrm{~mm} \mathrm{Hg}$, pulse was $60 / \mathrm{min}$ and rhythmic, and sclera was sub-icteric. The other systemic physical examinations were normal. The laboratory results were as follows: sedimentation: $32 \mathrm{~mm} / \mathrm{h}$, all hepatic serology tests were negative, aPTT: 31 s, PTT: 12 s, INR: 0.83, ANA: (++), anti-dsDNA: (-), AMA: (-), cANCA: (-), pANCA: (-) and TRAbs: (-). The thyroid Doppler-ultrasonography showed that both thyroid lobes were normal size with lobulated borders, excessive heterogenic and decreased echogenic parenchyma, and minimally 
Table 1. The Laboratory Values Before, During and After the Methimazole Treatment

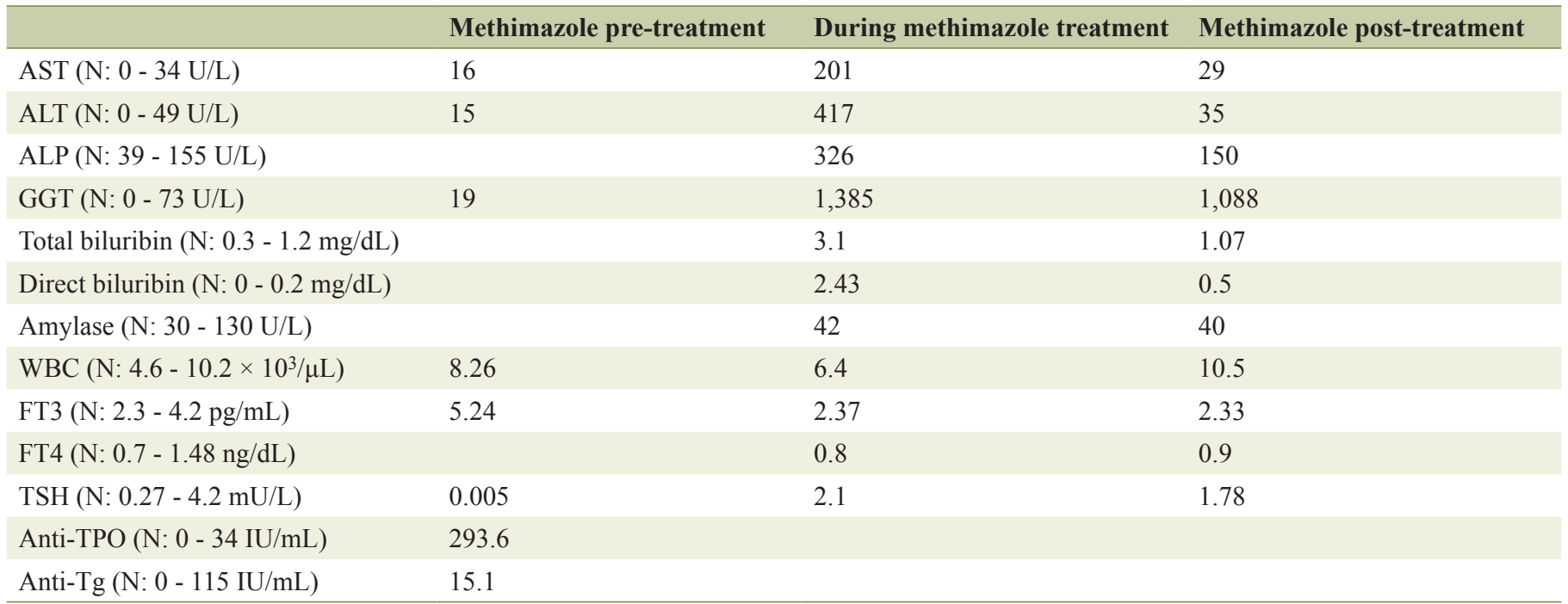

increased vascularity. On thyroid scintigraphy taken $20 \mathrm{~min}$ after intravenous administration of $4 \mathrm{mCi}$ Tc-99m pertechnetate, thyroid gland localization was normal. Activity distribution was relatively homogeneous. Uptake was increased visually. Our differential diagnosis included autoimmune thyroiditis and drug-induced hepatotoxicity. For this reason, methimazol treatment was stopped. Patient's liver enzyme values turned to normal on follow-up within 15 days (Table 1). Patient who had no symptoms and normal thyroid hormone profile was advised to be followed up at outpatient clinic.

\section{Discussion}

Methimazole, a thioureylene anti-thyroid drug, is well-known adverse drug reactions such as teratogenicity, agranulocytosis, acute hepatic necrosis and cholestasis hepatitis. Drug-induced hepatotoxicity is difficult to diagnose, and some criteria are needed to make the diagnosis. In 1989, an international consensus was carried out at Paris and some criteria were introduced. Nowadays, CIOMS/RUCAM scale (Council for International Organizations of Medical Sciences/Roussel Uclof Causolity Assessment Method) is used for assessment (Table 2) [4]. Hung et al [5] documented a case of hepatotoxicity induced by methimazole treatment after 5 months of methimazole treatment. In our patient, it was 1 month after the beginning of methimazole treatment. The presences of risk factors for other liver diseases were excluded with clinical history and physical examination in our patient. The presented case had 11 points which was calculated using CIOMS scale, which suggested

Table 2. CIOMS/RUCAM Scale

\begin{tabular}{llll}
\hline Type of liver injury & Hepatocellular & Cholestatic/mixed & Points \\
\hline $\begin{array}{l}\text { Time of onset of the event } \\
\text { Time from drug intake }\end{array}$ & First exposure & Second exposure & First exposure \\
until reaction onset & $5-90$ days & $1-15$ days & $5-90$ days \\
$\begin{array}{l}\text { Time from drug withdrawal } \\
\text { until reaction onset }\end{array}$ & $\leq 5$ or $>90$ days & $>15$ days & $\leq 5$ or $>90$ days \\
Risk factors & Alcohol & $\leq 15$ days & $\leq 30$ days \\
& Age $\geq 55$ years & Alcohol or pregnancy & +1 \\
Course of the reaction & $>50 \%$ improvement 8 days & - & +1 \\
& $>50 \%$ improvement 30 days & $>50 \%$ improvement 180 days & +2 \\
& - & $<50 \%$ improvement 180 days & +1 \\
& Lack of information or & Lack of information or & +0 \\
\hline
\end{tabular}

Score analysis: 0 or lower: relationship with the drug excluded; 1 - 2: unlikely; 3 - 5: possible; 6 - 8: probable; > 8: highly probable. 
that methimazole-treatment-induced hepatotoxicity was high probability. The estimated incidence of hepatotoxicity induced by anti-thyroid drugs is $0.1-0.2 \%$; however, actual incidence is unknown. The $25-50 \%$ of fulminant hepatic failure cases can be fatal [2]. A review of the literature has revealed around 22 cases of cholestatic jaundice due to both methimazole and carbimazole treatments [6]. Thionamide-induced liver damage is an idiosyncratic reaction that can develop at any time, but usually occurs within the first 3 months of treatment. It occurs in about $1 \%$ of patients, with a predisposition for women younger than 30 years of age [7]. Methimazole treatment usually causes cholestatic type hepatotoxicity. Mechanism of hepatic injury caused by methimazole is not well known, but an immune mediated injury may play a role [8]. It is important to follow the patient for the development of hepatic injury which may occur during the treatment with methimazole, which is an important drug in hyperthyroidism treatment.

\section{References}

1. Braverman L, Utiger R. Introduction to thyrotoxicosis. In: Braverman L, Utiger R, eds. The Thyroid. 9th ed.
Philadelphia: Lippincott Williams \& Wilkins. 2005, pp. 453-455.

2. Cooper DS. The side effects of antithyroid drugs. Endocrinologist. 1999;457-476.

3. Shlomo M, Kenneth SPP, Reed L, Henry MK. Williams Textbook Endocrinology, 12th Edition. 2011, pp. 380381.

4. Andrade RJ, Robles M, Fernandez-Castaner A, LopezOrtega S, Lopez-Vega MC, Lucena MI. Assessment of drug-induced hepatotoxicity in clinical practice: a challenge for gastroenterologists. World J Gastroenterol. 2007;13(3):329-340.

5. Hung YT, Yu WK, Chow E. Delayed cholestatic hepatitis due to methimazole. Hong Kong Med J. 1999;5(2):200201.

6. Mikhail NE. Methimazole-induced cholestatic jaundice. South Med J. 2004;97(2):178-182.

7. Malik R, Hodgson H. The relationship between the thyroid gland and the liver. QJM. 2002;95(9):559-569.

8. Blom H, Stolk J, Schreuder HB, von Blomberg-van der Flier M. A case of carbimazole-induced intrahepatic cholestasis. An immune-mediated reaction? Arch Intern Med. 1985;145(8):1513-1515. 